D. Eisenbud and E. G. Evans, Jr.

Nagoya Math. J.

Vol. 62 (1976), 41-53

\title{
A GENERALIZED PRINCIPAL IDEAL THEOREM
}

\section{DAVID EISENBUD* AND E. GRAHAM EVANS, JR.*}

Krull's principal ideal theorm [Krull] states that $q$ elements in the maximal ideal of a local noetherian ring generate an ideal whose minimal components are all of height at most $q$. Writing $R$ for the ring, we may consider the $q$ elements, $x_{1}, \cdots, x_{q}$ say, as coordinates of an element $x \in R^{q}$. It is an easy observation that every homomorphism $R^{q} \rightarrow R$ carries $x$ to an element of the ideal generated by $x_{i}, \cdots, x_{q}$. Write $R^{q^{*}}$ for $\operatorname{Hom}_{R}\left(R^{q}, R\right)$ and set

$$
R^{q^{*}}(x)=\left\{\varphi(x) \mid \varphi \in \operatorname{Hom}\left(R^{q}, R\right)\right\}
$$

We may re-express Krull's theorem as saying that, if $J$ is the maximal ideal of $R$, and $x \in J R^{q}$, then the minimal components of $R^{q^{*}}(x)$ have height at most $q$. In fact by localizing at an arbitrary minimal prime of $R^{q^{*}}(x)$, we see that it is enough merely to say that the height of $R^{q^{*}}(x)$-that is, the minimum of the heights of the primes containing $R^{q^{*}}$-is at most $q$.

In this paper we will generalize this re-statement of Krull's theorem -in the case in which $R$ has Cohen-Macaulay modules-by replacing $R^{q}$ with an arbitrary finitely generated module of rank $q$ (see Section 1 for definitions). (Hochster has proved $[\mathrm{H}]$ that every local ring which contains a field does in fact have Cohen-Macaulay modules (as does any Cohen-Macaulay ring). He further conjectures that every local ring has them.)

Special cases of Krull's theorem (for polynomial rings) were known already to Kronecker [Kron, p. 80], and in this setting the theorem was generalized by Macaulay in 1916 ([Mac], $\S \S 47-53$ ) to ideals of minors of matrices (a minor is the determinant of a submatrix): He showed that, if $\varphi$ is an $s \times t$ matrix over $R$, with $s \leq t$, say, and if the ideal

\footnotetext{
Received October 29, 1975.

* Both authors wish to acknowledge the support of the Alfred P. Sloan Foundation and the NSF.
} 
of $s \times s$ minors of $\varphi$ is contained in $J$, then all the minimal primes of that ideal have height at most $t-s+1$ (Krull's theorem is the case $s=1, t=q$ ). This was extended to general commutative rings by Northcott $[N]$, and to lower order minors by Eagon [Ea]. Writing $I_{k}(\varphi)$ for the ideal generated by the $k \times k$ minors of $\varphi$, Eagon's theorem says that if $I_{k}(\varphi) \subset J$, then the height of $I_{k}(\varphi)$ is at most $(s-k+1)(t-k+1)$. (A very elegant proof of this is given in [E $-\mathrm{N}$, section 6].)

Our generalization of Krull's theorem contains these generalizations, and allows us to prove somewhat more; for example, we show that if the entries of the matrix $\varphi$ are contained in $J$, and if the height of $I_{k}(\varphi)$ is $(s-k+1)(t-k+1)$, the largest possible, then for every submatrix $\psi$ of $\varphi$, the ideal $I_{k}(\psi)$ is also, in a certain sense, as large as possible. In particular, no $k \times k$ minor of $\varphi$ is zero.

A generalization of Krull's theorem in a different direction from ours is contained in intersection theory. Serre's Intersection Theorem [S, V, Theorem 3] says that if $R$ is a regular local ring, and if $I_{1}$ and $I_{2}$ are ideals of $R$, then

$$
\text { height }\left(I_{1}+I_{2}\right) \leq \text { height } I_{1}+\text { height } I_{2} \text {. }
$$

Krull's theorem, and the theorems of Macaulay, Northcott, and Eagon follow easily; Serre's theorem is equivalent to the statement that if $R$ $\rightarrow S$ is a map of noetherian rings, with $R$ regular, and if $I$ is a prime ideal of $R$, then height $I S \leq$ height $I$, so the heights of ideals generated by $n$ elements, or ideals of minors, are bounded by the heights of the "generic examples" of these sorts of ideals, which are easy to compute directly. Similarly, Peskine-Szpiro's "Intersection Conjecture" (proved in many cases in $[\mathrm{P}-\mathrm{S}]$, and generalized to the case of rings with Cohen-Macaulay modules in $[H]$ ) bounds the height of $I_{1}+I_{2}$, in a way depending on the projective dimension of $R / I_{1}$, without assuming that $R$ is regular.

Our generalization of the Principal Ideal Theorem yields another "intersection theorem" of this sort. One version of our theorem, Corollary 1.2, says that if $R$ is a local ring (with Cohen-Macaulay modules), and if $\varphi: M \rightarrow R$ is an element of $J M^{*}$, then

$$
\text { height } \varphi(M) \leq \operatorname{rank} M \text {. }
$$

Thus if we define the $r$-height of an ideal $I$ by 


$$
r \text {-height } I=\min _{\substack{\varphi: M \rightarrow M^{\prime} I \\ \varphi \in \mathcal{J M}^{*}}} \operatorname{rank} M
$$

we have height $I \leq r$-heignt $I$. But it is easy to see that $r$-height is subadditive: $r$-height $\left(I_{1}+I_{2}\right) \leq r$-height $I_{1}+r$-height $I_{2}$, since if $\varphi_{1}: M_{1} \longrightarrow$ $I_{1}$ and $\varphi_{2}: M_{2} \longrightarrow I_{2}$, with $\varphi_{i} \in J M_{i}^{*}$, then $\varphi_{1}+\varphi_{2}: M_{1} \oplus M_{2} \longrightarrow I_{1}+I_{2}$ is in $J\left(M_{1} \oplus M_{2}\right)^{*}$, and $\operatorname{rank} M_{1} \oplus M_{2} \leq \operatorname{rank} M_{1}+\operatorname{rank} M_{2}$. Thus, by Corollary 1.2 we have

$$
\text { height } I_{1}+I_{2} \leq r \text {-height } I_{1}+r \text {-height } I_{2} .
$$

It now makes sense to ask for which ideals $I$ is $r$-height $I=$ height $I$ ? Of course, this is so if $I$ is generated by an $R$ sequence, and it is not too difficult to show that the equality holds for ideals of projective dimension 1 . In fact, we know of no examples of ideals of finite projective dimension for which $r$-height $\neq$ height. If there were none-even just for regular local rings-we would recover Serre's theorem.

We owe a debt of gratitude to David A. Buchsbaum, with whom we first conjectured the results of this paper (we were motivated by a desire to prove Corollary 2.4). We also profitted from discussions with Phillip A. Griffith and Melvin Hochster; in particular, the use of CohenMacaulay modules was Hochster's suggestion.

\section{§1. The generalized principal ideal theorem}

Throughout this section, $R$ will denote a noetherian local ring.

We begin by recalling some definitions.

The dimension of $R$ is maximum of the lengths of chains of prime ideals of $R$. The height of a prime ideal $P$ of $R$ is the dimension of $R_{P}$. The height of an arbitrary proper ideal $I$ of $R$ is the minimum of the heights of prime ideals containing $I$.

If the dimension of $R$ is $n$, then a system of parameters for $R$ is a set of $n$ elements which generate an ideal of height $n$. It is not difficult to see that any ideal of height $h$ contains $h$ elements of some system of parameters.

If $N$ is an $R$-module, then $x_{1}, \cdots, x_{n} \in R$ are said to be an $N$-sequence if

1) $x_{i+1}$ is a nonzerodivisor on $N /\left(x_{1}, \cdots, x_{i}\right) N$ for $0 \leq i<n$ and

2) $\left(x_{1}, \cdots, x_{n}\right) N \neq N$. 
We will say that $R$ has Cohen-Macaulay modules if for every system of parameters $\left\{x_{1}, \cdots, x_{n}\right\}$ of $R$, there exists an $R$-module $N$ (possibly not finitely generated) such that $x_{1}, \cdots, x_{n}$ is an $N$-sequence. Hochster has shown [H] that if $R$ contains a field, then $R$ does indeed have CohenMacaulay modules, and has conjectured that all local rings have them.

If $M$ is an $R$-module and $x \in M$ is an element, we write $M^{*}=\operatorname{Hom}_{R}(M, R)$ for the dual module, and define the order ideal of $x$ by

$$
M^{*}(x)=\left\{\varphi(x) \mid \varphi \in M^{*}\right\} .
$$

Finally, the rank of a finitely generated $R$-module $M$, is the maximum of the numbers

$$
\operatorname{dim}_{R_{P} / P R_{P}} M_{P} / P M_{P}
$$

where $P$ ranges over the minimal primes of $R$.

Note that this is the usual definition of rank in the case $R$ is a domain, but differs slightly from it in the general case (the "usual" rank is defined as the minimum instead of the maximum).

We are now ready to state our main result:

THEOREM 1.1. Let $R$ be a local ring with maximal ideal $J$, and suppose that $R$ has Cohen-Macaulay modules. Let $M$ be a finitely generated $R$-module, and let $x \in J M$ be an element. Then

$$
\text { rank } M \geq \text { height } M^{*}(x) \text {. }
$$

The theorem could easily be deduced from either of the following two results, which we will prove as corollaries:

COROLlaRY 1.2. With the hypotheses of the Theorem, suppose that $\varphi \in J M^{*}$. Then

$$
\operatorname{rank} M \geq \text { height } \varphi(M) \text {. }
$$

The next result allows one to conclude that an element of a module is part of a minimal system of generators from certain information about the localizations of the module at non-maximal primes:

COROLlaRY 1.3. With the hypotheses of the Theorem, suppose that $\operatorname{rank} M<\operatorname{dim} R$. If an element $y \in M$ is such that for every nonmaximal prime $P, y \in M_{P}$ generates a free summand of $M_{P}$, then $y$ is part of a minimal system of generators of $M$. 
Remarks: 1) Therem $\mathrm{A}$ of $[\mathrm{E}-\mathrm{E}]$ (or a theorem of $[\mathrm{B}-\mathrm{M}]$ ) shows that if $\operatorname{rank} M \geq \operatorname{dim} R$ and $M_{P}$ is free for all non-maximal primes $P$, then it is possible to find an element $y \in J M$-that is, an element which is not part of a minimal system of generators of $M$-which generates a free summand of $M_{P}$ for each $P$, so the bound $\operatorname{rank} M<\operatorname{dim} R$ is best possible.

2) To see that the hypothesis of Corollary 1.3 cannot be weakened to require only that $y \in M_{P}$ is part of a minimal system of generators for each non-maximal $P$, let $k$ be a field, and consider the ring

$$
R=k[s, t, u, v] .
$$

Let $M=(s, t) \oplus R \oplus R$; this is a module of rank 3. Then $y=(u s+$ $v t, s, t) \in M$ is clearly part of a minimal system of generators of $M_{P}$ unless $P=(s, t, u, v)$.

3) To obtain a theorem which is valid without the existence of Cohen-Macaulay modules we could have redefined the rank by taking the maximum of $\operatorname{dim}_{R_{P} / P R_{P}} M_{P} / P M_{P}$ over all the associated primes of 0 . The proof we will give for the theorem then shows that this possibly larger rank for $M$ bounds the depth of the ideal $M^{*}(x)$, or even its depth with respect to a finitely generated module $N$. Corollary 1.2, and some of the results on determinantal ideals given later on could also be treated in this way.

Proof of Theorem 1.1. We first reduce to the case in which $R$ is an integral domain. There is clearly a minimal prime $P$ of $R$ such that

$$
\text { height } M^{*}(x)=\operatorname{height}_{R / P}\left(M^{*}(x)+P\right) / P \text {. }
$$

Since homomorphisms $M \rightarrow R$ induce homomorphisms

$$
M \otimes R / P=M / P M \rightarrow R / P,
$$

we have $\left(M^{*}(x)+P\right) / P \subseteq(M / P M)^{*}(x)$. Because of the way rank $M$ was defined, we have

$$
\operatorname{rank}_{R / P} M / P M \leq \operatorname{rank} M .
$$

Thus, if we knew the theorem for the domain $R / P$, we would have

$$
\begin{aligned}
\operatorname{rank} M & \geq \operatorname{rank}_{R / P}(M / P M) \geq \operatorname{height}_{R / P}(M / P M)^{*}(x) \\
& \geq \operatorname{heignt}_{R / P}\left(M^{*}(x)+P\right) / P=\operatorname{height} M^{*}(x) .
\end{aligned}
$$


Henceforward, we will assume that $R$ is an integral domain.

We will next reduce to the case in which

$$
\text { height }\left(M^{*}(x)\right)=\operatorname{dim} R
$$

Suppose that height $M^{*}(x)=h<\operatorname{dim} R$, and that $x_{1}, \cdots, x_{n}$ is a system of parameters for $R$ of which the first $h$ are in $M^{*}(x)$. Let $x^{\prime} \in M \oplus R^{n-h}$ $=M^{\prime}$ be the element $\left(x, x_{h+1}, \cdots, x_{n}\right)$. Clearly $x^{\prime} \in J M^{\prime}, M^{\prime *}\left(x^{\prime}\right) \supseteq\left(x_{1}\right.$, $\left.\cdots, x_{n}\right)$, and $\operatorname{rank} M^{\prime}=\operatorname{rank} M+n-h$. Thus if

$$
\text { rank } M^{\prime} \geq \text { height } M^{\prime *}\left(x^{\prime}\right)=n,
$$

then $\operatorname{rank} M \geq h=$ height $M^{*}(x)$, and we may suppose that $M^{*}(x)$ already has height $n$.

Now let $a_{i} \in M^{*}$ be such that $x_{1}=a_{1}(x), \cdots, x_{n}=a_{n}(x)$ is a system of parameters for $R$ in $M^{*}(x)$, and let $I$ be the ideal generated by the $x_{i}$. Define a map $a: M \rightarrow R^{n}$ by $m \mapsto\left(a_{1}(m), \cdots, a_{n}(m)\right)$. Suppose that $y_{1}, \cdots, y_{m}$ generated the maximal ideal $J$ of $R$. Since $x \in J M$, there is a map $b: R^{m} \rightarrow M$ sending the vector $\left(y_{1}, \cdots, y_{m}\right)$ to $x$.

Define $f: R^{n^{*}} \rightarrow R^{m^{*}}$ as the composite

1)

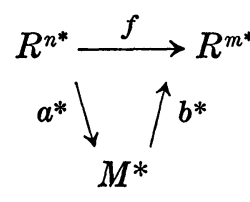

This $f$ fits into a commutative diagram

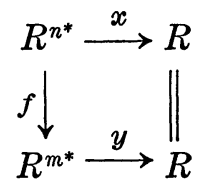

where $x$ and $y$ are given by the matrices $\left(x_{1}, \cdots, x_{n}\right)$ and $\left(y_{1}, \cdots, y_{m}\right)$ respectively. This diagram can be extended in the usual way to a comparison map of the Koszul complexes of $I$ and $J$ :

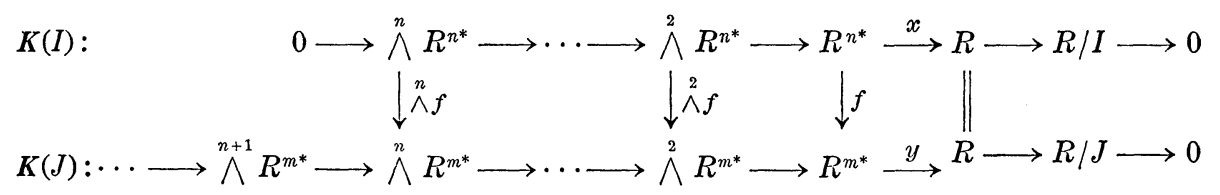


Suppose that, contrary to the theorem, $\operatorname{rank} M=r<n$. Then we claim $\bigwedge^{n} f=0$. Writing $R_{(0)}$ for the quotient field of $R$, we note that because $R$ is a domain and $\bigwedge^{n} f$ is a map of free $R$-modules, it suffices to check that $\left(\bigwedge^{n} f\right)_{(0)}=\left(\bigwedge^{n} f\right) \otimes R_{0}=0$. But this is the same as $\bigwedge_{R_{\text {; } 0)}}\left(f_{(0)}\right)$ $=0$, and the commutative diagram 1) shows that $\bigwedge_{R_{(0)}}^{n}\left(f_{(0)}\right)$ factors through $\bigwedge_{R_{(0)}}^{n}\left(M_{(0)}^{*}\right)=\bigwedge_{R_{(0)}}^{n}\left(M_{(0)}^{*}\right)$. Since $M_{(0)}$ is an $r$-dimensional $R_{(0)}$ vectorspace, and $r<n$, this module is clearly 0 , and $\wedge^{n} f=0$ as claimed.

It now suffices to show that $\bigwedge^{n} f \neq 0$ to establish the theorem. To do this we will examine the maps induced by $\wedge^{n} f$ on cohomology, using the following rather well-known lemma to shift the problem to a calculation of Ext.

LEMMA 1.4. Let $R$ be a noetherian ring, $I \subset R$ an ideal, and $K(I)$ the Koszul complex of $I$. There is a family of natural transformations from the cohomology of $\boldsymbol{K}(I)$ to $\operatorname{Ext}(R / I,-)$ :

$$
\alpha_{I}^{i}: H^{i}(\operatorname{Hom}(K(I),-)) \longrightarrow \operatorname{Ext}^{i}(R / I,-)
$$

such that

1) If $I \subset I^{\prime}$, then $\alpha$ commutes with the maps $\operatorname{Ext}\left(R / I^{\prime},-\right) \rightarrow \operatorname{Ext}(R / I,-)$ induced by the projection $R / I \rightarrow R / I^{\prime}$ and the maps induced on cohomology by any extension $K(I) \rightarrow K\left(I^{\prime}\right)$ of the inclusion $I \rightarrow I^{\prime}$, and

2) If $N$ is an $R$-module, and I contains an $N$-sequence of length $n$, then

$$
\alpha_{R / I, N}^{i}: H^{i}(\operatorname{Hom}(K(I), N)) \longrightarrow \operatorname{Ext}^{i}(R / I, N)
$$

is an isomorphism for $i \leq n$.

Sketch of proof of Lemma 1.4. $\alpha$ may be defined as the map on cohomology induced by any comparison map from the Koszul complex to a projective resolution of $R / I$. The homotopy-uniqueness of such a map yields the naturality, and part 1). Part 2) can be proved by induction on $i$, using the fact that both $\operatorname{Ext}(R / I,-)$ and the cohomology of $K(I)$ are connected sequences of functors. (See $[B-R]$ for a similar proof which is given in detail).

In order to apply the lemma, we choose an $R$-module $N$ such that 
$x_{1}, \cdots, x_{n}$ is an $N$-sequence. Since $\left(x_{1}, \cdots, x_{n}\right)=I \subset J, \operatorname{Ext}^{n}(R / I, N) \cong$ $\operatorname{Hom}(R / I, N / I N)$, and $\operatorname{Ext}^{n}(R / J, N) \cong \operatorname{Hom}(R / J, N / I N)$, both isomorphisms being natural. From this and Lemma 1.4, we obtain a commutative diagram

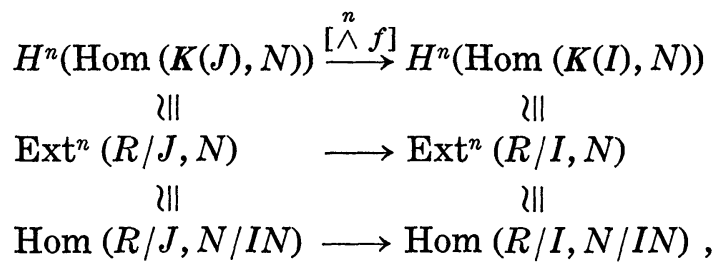

where $\left[\bigwedge^{n} f\right]$ denotes the map induced by $\bigwedge^{n} f$ on cohomology, and the map Hom $(R / J, N / I N) \rightarrow \operatorname{Hom}(R / I, N / I N)$ is induced by the natural projection $R / I \longrightarrow R / J$. Since the map from $R / I$ to $R / J$ is an epimorphism, the induced map on Hom is a monomorphism, and it suffices to show that

$$
\operatorname{Hom}(R / J, N / I N) \neq 0 \text {. }
$$

Since $I=\left(x_{1}, \cdots, x_{n}\right), N / I N \neq 0$, and since $x_{1}, \cdots, x_{n}$ is a system of parameters, $J^{k} \subset I$ for some $k$. It follows that $N / I N$ does contain nonzero elements annihilated by $J$, so $\operatorname{Hom}(R / J, N / I N) \neq 0$. Thus $\operatorname{rank} M \geq$ height $M^{*}(x)$, as claimed.

Proof of Corollary 1.2. As in the proof of the theorem, one reduces to the case in which $R$ is a domain. It follows that $M, M^{*}$, and $M^{* *}$ all have the same rank. Since $\varphi(M) \subset M^{* *}(\varphi)$, the Corallary follows from the Theorem, applied to the element $\varphi \in M^{*}$.

Proof of Corollary 1.3. Since the formation of $M^{*}(y)$ commutes with localization, we see that $M^{*}(y)$ is not contained in any prime ideal of $R$ except $J$; thus height $M^{*}(y)=\operatorname{dim} R$. By the Theorem, if $y \in J M$, we could conclude $\operatorname{rank} M \geq \operatorname{dim} R$, a contradiction.

\section{§2. Determinantal ideals}

Throughout this section $R$ will denote a local ring with maximal ideal $J$, and $\varphi$ will denote an $s \times t$ matrix with elements in $R$. We will assume throughout that $R$ has Cohen-Macaulay modules; (see note 3 after Theorem 1.1 for a suggestion of a generalization that works without this hypothesis). 
If $k$ is an integer, we write $I_{k}(\varphi)$ for the ideal of $k \times k$ minors of $\varphi$.

THEOREM 2.1. With notation as above, suppose that $I_{k}(\varphi)=0$, and let $\varphi^{\prime}$ be the $s \times(t+1)$ matrix obtained from $\varphi$ by adjoining a column with entries in $J$. Then

$$
\text { height } I_{k}\left(\varphi^{\prime}\right) \leq s-k+1 \text {. }
$$

We will postpone the proof until later.

EXAMPLE 2.2. To see that the condition on the elements of the added column is necessary, consider the ring $R=k[[x, y]]$, and the matrices

$$
\varphi=\left(\begin{array}{ll}
0 & 0 \\
x & y
\end{array}\right) \quad \varphi^{\prime}=\left(\begin{array}{lll}
0 & 0 & 1 \\
x & y & 0
\end{array}\right) .
$$

Here $s=2=t$, and with $k=2$ we have $I_{k}(\varphi)=0$, but $I_{2}\left(\varphi^{\prime}\right)=(x, y)$ has height $2>s-k+1=1$.

The next Corollary is the now-classical formula for the heights of determinantal ideals.

COROLLARY 2.3 (Eagon-Macaulay-Northcott). With notation as above,

$$
\text { ht } I_{k}(\varphi) \leq(s-k+1)(t-k+1)
$$

Proof of Corollary 2.3. We induct on $s$ and $t$. Localizing, we may assume ht $I_{k}(\varphi)=\operatorname{dim} R$.

If some element of $\varphi$ is a unit, we may make a "change of basis" until $\varphi$ has the form

$$
\left(\begin{array}{c|c}
1 & 0 \cdots 0 \\
0 & \\
\vdots & \varphi^{\prime} \\
0 &
\end{array}\right)
$$

where $\varphi^{\prime}$ is an $(s-1) \times(t-1)$ matrix. Clearly $I_{k}(\varphi)=I_{k-1}\left(\varphi^{\prime}\right)$, and we are done by induction. Thus we may assume that all the elements of $\varphi$ belong to $J$.

Next, by completing $R$ and factoring out a minimal prime ideal, we may assume that $R$ is an integral domain with saturated chain con- 
dition-that is, for every prime $P$ of $R$, height $P+\operatorname{dim} R / P=\operatorname{dim} R$.

Finally, consider an $s \times(t-1)$ submatrix $\psi$ of $\varphi$. By induction, height $I_{k}(\psi) \leq(s-k+1)(t-k)$. Let $P$ be a prime containing $I_{k}(\psi)$ with the same height. By Theorem 2.1, $\operatorname{dim} R / P=\operatorname{height}_{R / P}\left(I_{k}(\varphi)+P\right) / P$ $\leq s-k+1$. By the saturated chain condition,

$$
\text { height } \begin{aligned}
I_{k} \varphi & =\operatorname{dim} R \\
& =\operatorname{height} P+\operatorname{dim} R / P \\
& \leq(s-k+1)(t-k)+(s-k+1) \\
& =(s-k+1)(t-k+1),
\end{aligned}
$$

as claimed.

Remark. This proof, with its reliance on Cohen-Macaulay modules and the saturated chain condition, is not to be taken too seriously; there is an elementary and beautiful proof of the same fact in $[\mathrm{E}-\mathrm{N}$, section 6]. We include it because it seems amusing to note that Theorem 1.1 "contains" the other "generalized principal ideal theorem."

Our next result is a sort of rigidity formula, of the type that says that any subset of an $R$-sequence is an $R$-sequence.

Since we are working with height such statements are slightly treacherous; it is not true for example that if $n$ elements generate an ideal of height $n$, then any subset of $k$ elements generates an ideal of height $k$. For example let $R=\frac{k[x, y, z]}{(x, y) \cap(z)}$, where $k$ is a field. Then the ideal $(x, y+z)$ has height 2 , but $(x)$ has height 0 .

To avoid this sort of difficulty, we work instead with dimension; it is true that if

$$
\operatorname{dim} R /\left(x_{1}, \cdots, x_{n}\right)=(\operatorname{dim} R)-n
$$

then $\operatorname{dim} R /\left(x_{1}, \cdots, x_{k}\right)=(\operatorname{dim} R)-k$, for any $k$. Of course if one sticks to the case of an equidimensional ring with saturated chain conditionnot a very drastic restriction-one recovers statements about height. Also, the reader will note that if $h t I=n$, then $\operatorname{dim} R / I \leq(\operatorname{dim} R)-n$ in any case. Our main result of this type is:

COROLlaRY 2.4. Suppose as before that $\varphi$ is an $s \times t$ matrix with 
coefficients in $J$, and let $\psi$ be a submatrix of size $u \times v$, say. If, for some $k$,

$$
\operatorname{dim}\left(R / I_{k}(\varphi)\right) \leq(\operatorname{dim} R)-(s-k+1)(t-k+1)
$$

then

$$
\operatorname{dim}\left(R / I_{k}(\psi)\right) \leq(\operatorname{dim} R)-(u-k+1)(v-k+1) .
$$

Remark. Example 2.2 shows again that the hypothesis that the hypothesis that the coefficients of $\varphi$ are in $J$ is essential.

CoRollary 2.5. If $\varphi$ is an $s \times t$ matrix with coefficients in $J$ and for some $k$

$$
\text { height } I_{k}(\varphi)=(s-k+1)(t-k+1),
$$

then no $k \times k$ minor of $\varphi$ is 0 .

Corollary 2.5 is an immediate consequence of Corollary 2.4.

Remark. The following conjecture, if true, would allow one to strengthen the conclusion of Corollaries 2.4 and 2.5 to statements about ideals of minors of any size $\geq k$.

CONJECTURE 2.6. If $\varphi$ is an $s \times t$ matrix (not necessarily with coefficients in the maximal ideal of $R$ ) such that $I_{k+1}\left(\varphi^{\prime}\right)=0$, then $h t I_{k}(\varphi)$ $\leq s+t-2 k+1$.

Proof of Corollary 2.4. We will prove the corollary in the case $u$ $=s, v=t-1$; an iterated use of this (and the version interchanging $u$ and $v$ ) produces the general result.

Let $\hat{R}$ be the completion of $R$. For any ideal $I$ of $R, \operatorname{dim} R / I=$ $\operatorname{dim} \hat{R} / I \hat{R}$. Since

$$
\operatorname{dim} R / I_{k}(\psi)=\operatorname{dim} \hat{R} / I_{k}(\psi) \hat{R},
$$

there exists a minimal prime ideal $P$ of $\hat{R}$ containing $I_{k}(\psi)$ such that $\operatorname{dim} \hat{R} / P=\operatorname{dim} R / I_{k}(\psi)$, and since

$$
\operatorname{dim} \hat{R} / P+I_{k}(\varphi) \leq \operatorname{dim} \hat{R} / I_{k}(\varphi)=\operatorname{dim} R / I_{k}(\varphi),
$$

we may assume $R=\hat{R} / P$.

In particular, we may assume that $R$ is an integral domain with saturated chain condition, and $I_{k}(\psi)=0$. Under these circumstances 


$$
\operatorname{dim} R / I_{k}(\psi)=\operatorname{dim} R=\operatorname{dim} R / I_{k}(\varphi)+\text { height } I_{k}(\varphi),
$$

so the Corollary follows from Theorem 2.1.

Proof of Theorem 2.1. As usual, we may assume that $R$ is a domain, and $k>1$. Because of the Laplace expansion of $k \times k$ minors of $\psi$ involving the last column along the last column, we may assume $I_{k-1}(\varphi)$ $\neq 0$.

We now regard $\varphi$ as a map $R^{t} \rightarrow R^{s}$, and we set $M=\operatorname{coker} \varphi$. Because $I_{k-1}(\varphi) \neq 0$ and $I_{k}(\varphi)=0$, we have $\operatorname{rank} M=s-k+1$. Let $\bar{x}$ be the image in $M$ of the new column $x$ of $\varphi^{\prime}$, regarded as an element of $R^{s}$. Since $x \in J R^{s}$, we have $\bar{x} \in J M$. By Theorem 1.1, height $M^{*}(\bar{x}) \leq s$ $-k+1$. We will show that any prime containing $M^{*}(\bar{x})$ must contain $I_{k}(\psi)$, from which height $I_{k}(\psi) \leq$ height $M^{*}(\bar{x})$ follows.

Suppose, then, that a prime ideal $P$ fails to contain $I_{k}(\psi)$. Localizing at $P$, we have

$$
\begin{aligned}
R_{P} & =I_{k}(\psi)_{P}=I_{k-1}(\varphi)_{P} \\
0 & =I_{k}(\varphi)_{P}=I_{k+1}(\psi)_{P} .
\end{aligned}
$$

Since $\psi$, thought of as a map $R^{t+1} \rightarrow R^{s}$, has cokernel $M / R \bar{x}$ it follows by an easy argument from $*$ ) that $M_{P}$ and $(M / R \bar{x})_{P}$ are both free, of ranks $s-k+1$ and $s-k$, respectively (see, for example, [B $-\mathrm{E}$, lemma 1]). Thus $\bar{x}$ generates a free summand of $M_{P}$, so $M^{*}(\bar{x})_{P}=$ $\left(M_{P}\right) *(\bar{x})=R_{P}$. That is, $M^{*}(\bar{x}) \varnothing P$, as required.

Remark. It is also easy to exhibit the maps $M \rightarrow R$ which take $\bar{x}$ to the $k \times k$ minors of $\psi$ containing the last column. For, if $\alpha \in \bigwedge^{k-1} R^{t^{*}}$ $\otimes \bigwedge^{k-1} R^{s}$ corresponds under the isomorphism

$$
\bigwedge^{k-1} R^{t^{*}} \otimes \bigwedge^{k-1} R^{s} \cong \operatorname{Hom}_{R}\left(\bigwedge^{k-1} R^{t}, \bigwedge^{k-1} R^{s}\right)
$$

to the map $\bigwedge^{k-1} \varphi$, then the map "multiplication by $\alpha$ "

$$
m_{\alpha}: R^{s} \longrightarrow \bigwedge^{k-1} R^{t^{*}} \otimes \bigwedge^{k} R^{s}: x \longmapsto a \wedge x
$$

satisfies $m_{\alpha} \varphi=0$ since $\bigwedge^{k} \varphi=0$, and thus induces a map

$$
\bar{m}_{\alpha}: M \longrightarrow \bigwedge^{k-1} R^{t^{*}} \otimes \bigwedge^{k} R^{s}
$$

The various coordinate projections to $R$ of $\bar{m}_{\alpha}(\bar{x})=m_{\alpha}(x)$ give the required $k \times k$ minors of $\psi$. 


\section{REFERENCES}

[B-M] Bass, H. and Murthy, M. P.: Grothendieck Groups and Picard Groups of Abelian Group Rings, Ann. of Math. 86 (1967), 16-73.

[B-E] Buchsbaum, D. A. and Eisenbud, D.: What makes a complex exact? J. Alg. 13 (1973), 259-268.

[B-R] Buchsbaum, D. A. and Rim, D. S.: A generalized Koszul Complex II-Depth and Multiplicity, Trans. Am. Math. Soc. 111 (1964), 197-225.

[Ea] Eagon, J. A.: Ideals generated by subdeterminants of a matrix, Thesis, University of Chicago (1961).

[E-N] Eagon, J. A. and Northcott, D. G.: Ideals defined by matrices and a certain complex associated with them, Proceedings of the Royal Soc. of England A, 269 (1962), 188-204.

[E-E] Eisenbud, D. and Evans, E. G.: Generating modules efficiently; Theorems from algebraic K-theory, J. Alg. 27 (1973), 278-305.

[H] Hochster, M.: Deep local rings, to appear.

[Kron] Kronecker, L.: Grundzüge einer arithmetischer Theorie der algebraischen Grössen, J. Reine angew. Math. 92 (1882), 1-122.

[Krull] Krull, W.: Über einen Hauptsatz der allgemeinen Idealtheorie, S.-B. Heidelberg Akad. Wiss. (1929), 11-16.

[Mac] Macaulay, F. S.: The Algebraic Theory of Modular Systems, Cambridge Tracts in Mathematics and Math. Physics, 19 (1916).

[N] Northcott, D. G.: Semi-regular rings and semi-regular ideals, Quart. J. Math. (2) 11 (1960), 81-104.

[P-S] Peskine, C. and Szpiro, L.: Dimension projective finie et cohomologie locale, Pub. I.H.E.S. Paris 42, 1973.

[S] Serre, J.-P.: Algèbre Locale-Multiplicités, Springer Lecture Notes in Math. $11,1958$.

Dept. of Math.

Brandeis University

Waltham Ma. 02166

USA

Dept. of Math.

University of Illinois

Urbana, Ill. 61801 